\title{
Parametric optimization of the configuration and operation of transparent and opaque climate responsive building facade modules using component scale simulation
}

\author{
Shahrzad Soudian ${ }^{1 *}$, Umberto Berardi $^{2}$ \\ ${ }^{1}$ Mechanical and Industrial Engineering, Ryerson University, Toronto, Canada \\ ${ }^{2}$ Department of Architectural Science, Ryerson University, Toronto, Canada \\ *shahrzad.soudian@ryerson.ca
}

\begin{abstract}
The rising demand for energy-efficient building design is correlated with the negative effects of energy consumption in buildings on the environment, in addition to climate-related threats to their resiliency. Considering the highest share of energy use in buildings allocated to space conditioning, comfort-related energy-efficiency measures are primarily targeted. Particularly enhancing the performance of the building facade as the main interface between the interior and exterior environments has been a key research area to provide indoor environmental comfort. Conventionally, building facades are designed as static barriers to environmental loads, disregarding the transient nature of their surrounding environment. However, there is a need for a new paradigm in façade design to improve comfort and resiliency in buildings. Climate responsive facades (CRFs) are a potential solution to address comfort in buildings through dynamic and adaptive performance. The aim of this paper is to select the best configuration and operation strategy for one transparent and one opaque CRF module in the climate context of Toronto using parametric optimization analysis. The CRF modules were initially designed using a performance-based design framework in consideration of indoor comfort, climate, and façade performance metrics. The initial design of the modules is based on a ventilated cavity Trombe wall system. The opaque CRF module was designed to dynamically regulate temperature, airflow, and moisture loads, and the transparent CRF module controls solar radiation, temperature, moisture, and airflow. The two modules are modeled in the climate of Toronto, facing the south orientation in COMSOL Multiphysics $\mathbb{C}$ using the heat transfer and the CFD tools. The parametric optimization analysis covers both design and operation parameters. The design parameters defined are based on façade performance metrics, including material thermophysical properties, geometry, and configuration of the materials and systems. The operation parameters based on the indoor comfort requirements include the mechanical ventilation inputs, material properties for passive smart materials, and operation of active integrated heating systems. The range of values for the parameters is determined by the technical properties of the selected technologies. The façade configuration with the best performance annually is selected as the best option. The performance is benchmarked for heat exchange efficiency, heat transfer rate, airflow rate, temperature/moisture gradient, daylight transmission, and time lag. Design optimization using the simulation study allowed for an accurate selection and optimization of the best CRF module configuration. Notably, the results of this study could show the role of component scale simulation tools to design CRFs.
\end{abstract}

